\title{
External stenting of saphenous vein bypass grafts does not affect intraoperative transit-time flow measurement
}

\author{
Burak Can Depboylu1, Patrick O Myers ${ }^{1}$, Mootoosamy Parmeseeven ${ }^{1 *}$, Jalal Jolou', Kamran Ahmadov', \\ Saziye Karaca', Marck Licker ${ }^{2}$, Afksendiyos Kalangos', Mustafa Cikirikcioglu ${ }^{1}$ \\ From World Society of Cardiothoracic Surgeons 25th Anniversary Congress, Edinburgh \\ Edinburgh, UK. 19-22 September 2015
}

\section{Background/Introduction}

Saphenous vein grafts (SVG) are the most commonly used conduits for coronary artery bypass operations (CABG), despite their sub-optimal long-term patency. External stenting of SVG (eSVS ${ }^{\circledR}$ mesh) was recently proposed to improve their long term patency. Transit time flow measurement (TTFM) is a well described method for intraoperative quality control for CABG.

\section{Aims/Objectives}

The aim of this study is to assess whether external stenting of SVG affects perioperative TTFM.

\section{Methods}

Twenty six patients who underwent elective CABG were divided into two groups based usage of externally stented
SVG $\left(e^{\text {eSVS }}{ }^{\circledR}\right.$ mesh, $\left.\mathrm{n}=13\right)$, or bare SVG $(\mathrm{n}=13)$. The anastomotic quality were evaluated with TTFM using the Medi-Stim VeriQ flowmeter and a $4 \mathrm{~mm}$ probe. Perioperative data were given as median ( $\min$ - max) and compared between groups (Table 1).

\section{Results}

There was no significant difference between two groups regarding pre and peri-operative parameters, although more patients in the eSVS ${ }^{\circledR}$ mesh group had concomitant procedures $(3,23 \%$ vs. $2,15 \%, P>0.99)$. All SVG were patent in both groups at the end of the surgical procedure and TTFM values were similar. eSVS ${ }^{\circledR}$ mesh group had a trend for longer cardiopulmonary and aortic cross clamping times, which didn't reach statistical significance.

Table 1

\begin{tabular}{|c|c|c|c|c|c|}
\hline & \multicolumn{2}{|c|}{ Mesh covered SVG $(n=13)$} & \multicolumn{2}{|c|}{ Bare SVG $(n=13)$} & \multirow[t]{2}{*}{$P$} \\
\hline & median & $\min -\max$ & median & $\min -\max$ & \\
\hline Age (years) & 64 & $51-82$ & 64 & $59-80$ & $>0.05^{*}$ \\
\hline Bypass grafts & 3 & $1-4$ & 3 & $2-4$ & $>0.05^{*}$ \\
\hline Simultaneous concomitant operation & $3(23 \%)$ & & $2(15 \%)$ & & $>0.05^{\circ}$ \\
\hline CBP time (min) & 112 & $57-161$ & 94 & $52-134$ & $>0.05^{*}$ \\
\hline Cross Clamp Time (min) & 69 & $34-122$ & 63 & $28-96$ & $>0.05^{*}$ \\
\hline TTFM (ml/min) & 59 & 19-106 & 43 & $30-155$ & $>0.05^{*}$ \\
\hline $\mathrm{Pl}$ & 1.9 & $1.2-4.9$ & 2.3 & $1.3-2.9$ & $>0.05^{*}$ \\
\hline
\end{tabular}

*Mann-Whitney U Test, ${ }^{\circ}$ Fisher's exact test 


\section{Conclusion}

External stenting of SVG by eSVS ${ }^{\circledR}$ mesh does not extend the operative times. All SVG showed excellent flow and eSVS $^{\circledR}$ mesh coverage didn't impede TTFM or provide graft flow different to controls.

\section{Authors' details}

'Division of Cardiovascular Surgery, University Hospitals and Medical Faculty of Geneva, Geneva, Geneva, 1211, Switzerland. 'Division of Anaesthesiology, University Hospitals and Medical Faculty of Geneva, Geneva, Geneva, 1211, Switzerland.

Published: 16 December 2015

doi:10.1186/1749-8090-10-S1-A297

Cite this article as: Depboylu et al:: External stenting of saphenous vein bypass grafts does not affect intraoperative transit-time flow measurement. Journal of Cardiothoracic Surgery 2015 10(Suppl 1):A297.

Submit your next manuscript to BioMed Central and take full advantage of:

- Convenient online submission

- Thorough peer review

- No space constraints or color figure charges

- Immediate publication on acceptance

- Inclusion in PubMed, CAS, Scopus and Google Scholar

- Research which is freely available for redistribution

Submit your manuscript at www.biomedcentral.com/submit 